INTERNATIONAL DESIGN CONFERENCE - DESIGN 2018

https://doi.org/10.21278/idc.2018.0492

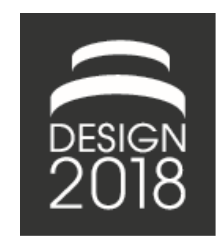

\title{
TOP-DOWN DESIGN COMPUTING BASED ON NON-MANIFOLD POLYHEDRA FROM FUNCTION-TO-FORM MAPPING
}

\author{
Z.-G. Xu, J.-F. Zhu, K.-Y. Su and W.-M. Liu
}

\begin{abstract}
In this paper, our research works are reported from requirement management to functional design, from automatic function-to-form mapping to shape decomposition and synthesizing, from traditional AI technologies to geometric reasoning etc. It is concluded that, management of creative process is the key issue to develop creative computational design tools; and the modelling of design tools could facilitate the creative thought processes. Several packages of design tools have been developed to testify creativity in design practices, and the relationship between creativity and automation.
\end{abstract}

Keywords: conceptual design, computational design methods, design creativity

\section{Introduction}

Digital design technology has been developed for more than half a century, from the early twodimensional wire-frame model, three-dimensional solid model, feature-modelling technology to the current intelligent, web-based design. Since the early stages of the CAD technology in 50s, the development trend of integration and standardization has formed the research category of the popular two-dimensional parameterization CAD system and the three-dimensional parameterized CAD system, where B-rep model, CSG method and feature technologies are widely utilized.

The design process is thought of being in several sequential phases of requirement acquisition, conceptual design, layout design, embodiment design and detailed design.

The recent researches are extending from functional design (Crocker and Reinke, 1991) to requirement management (Darrell, 2003), the mapping of requirement-to-function (Dietz et al., 1998), and functionto-form (El-Mehalawi and Miller, 2003). So far, computer-based design tools support design routine activities, such as documentation, storage, and translation of the design results. The attention of contemporary design applications is on the later phases of the design process, while the early phases are still poorly automated and receive little information support (Faltings and Sun, 1996).

Just as Yazdani (1994) have shown that, up till now no principal breakthrough has come about in applying computer-aided design tools in conceptual design phase. There are reasons to think that the absence of a design theory, which would coherently explicate the whole design process in a scientific way, is the main predicament for the development of more sophisticated computer tools capable of assisting human designers in their non-routine activities (Feng et al., 1996).

A top-down design computing methodology is studied in this paper, a D-M-R model is proposed for conceptual design based on the Non-manifold polyhedral from the function-to-form mapping.

So this paper is arranged in five sections.

Section 1 is the introduction to the paper;

Section 2 talked about the concept of the Non-manifold polyhedra from the function-to-form mapping; 
Section 3 talked about the D-M-R model and the design process with related to the D-M-R model;

Section 4 talked about the reconstitution of NMP;

Section 5 give one design example based on the proposed D-M-R model;

Section 6 discussed the proposed D-M-R model;

Section 7 draw conclusions for the paper.

\section{Non-manifold polyhedra from the function-to-form mapping}

A manifold model is called a "simple polyhedron", referred to a closed entity surrounded by a finite number of surfaces in R3 space, each side of a polyhedron has only two adjacent faces, all surfaces of a polyhedron can be traversed by crossing a common side from one side to another.

In the topological sense, the surface is a closed ring; any one edge is shared by two faces; and there are no isolated vertices.

All edges, rings, surfaces are fully defined in a manifold model. Geometric and topological validity of a manifold polyhedron satisfies the Euler formula:

$\mathrm{V}-\mathrm{E}+\mathrm{F}=2$

where, $\mathrm{V}$ means number of vertices, $\mathrm{E}$ means number of edges, $\mathrm{F}$ means number of faces.

Any "polyhedra" which does not satisfy the Euler formula, is called a non-manifold polyhedron, NMP for short.

From the view point of 3D conceptual structure design of mechanical products, in this paper the concept of NMP from function-to-form mapping is put forward, called FF-NMP.

FF-NMP is a cluster of conceptual features meeting for the transmission/positioning requirements. FF-NMP express the results of the intermediate and transitional properties of 3D conceptual design. According to different development stages, conceptual faces are hierarchically defined in Table 1.

Table 1. Hierarchical definitions of conceptual faces

\begin{tabular}{|l|l|}
\hline Conceptual face: Fi.type & \multicolumn{1}{c|}{ Concept and definition } \\
\hline Directional face DC & Described by outer vector (or axis of symmetry), a kind of limited face \\
\hline Oriented face OC & $\begin{array}{l}\text { Described by outer vector (or axis of symmetry) and a positioning point, a kind } \\
\text { of limited faces }\end{array}$ \\
\hline Qualitative face QC & $\begin{array}{l}\text { Described by outer vector (or axis of symmetry), a positioning point and a } \\
\text { functional area. }\end{array}$ \\
\hline Boundary face BC & Has the geometric information of boundary, vertex, edge, loop etc \\
\hline Closed face CC & Traditional displaying face \\
\hline
\end{tabular}

Conceptual Solid: A solid is composed of a group of conceptual faces $\mathrm{Fi}, \mathrm{i}=1,2, . . \mathrm{n}$, and/or position correlations, denoted by S.

Closeness of Conceptual Solid: For the Conceptual Solid S, if the stretched Fi, i=0,1,..n, under a certain positioning condition, could constitute a $3 \mathrm{D}$ closed space, then $\mathrm{S}$ is called closed. A closed solid is denoted by $\mathrm{G}$, which is called a complete bond graph.

If sub-graph, $\mathrm{Gi}$ is the graph of related conceptual faces belonging to $\mathrm{G}, \widetilde{G}_{i}$ is a set of unrelated functional faces, then $\mathrm{S} \subset\left(\mathrm{Gi}, \widetilde{G}_{i}\right)$. Symbol S: Fi.Type means, $\mathrm{S}$ is composed of the functional faces of the type Fi.Type, Fi.Type $\subset$ (DC, OC, QC, BC, CC) (from Table 1). For example, Gi:DC means the conceptual solid is composed of correlated DCs, while $\widetilde{G}_{i: Q C}$ means the solid being composed of unrelated QCs.

Abstract Feature: A special region belonging to a component to express functional information and geometric implication. It beholds either abstract information group composed of a number of faces and/or their relationships belonging to the domain of a conceptual solid, or accurate engineering semantics within design, manufacture, assembly and disassembly domains. 
Zero Solid (Zsolid) is proposed based on the following considerations:

1. Zero height: The functional face is regarded as an object with zero height, and it could be variation in size and shape;

2. Information minimization: The initial information contained in a conceptual part is minimized based on the direct and simple mapping;

3. Information independence: There is no function overlapping between Zsolids, and no redundant information;

Zsolid means variation in solid size, boundary, shape and topological information within the conceptual solid domain.

Conceptual part is defined as a network of Zsolids, denoted by ZsolidNet. ZsolidNet actually uses the data structure of object class list, and the associated function surface and the concave convex relation between function surface are stored in the form of double linked list, like in Figure 1.

The above-mentioned concepts, like conceptual solids and Zsolids are all belongs to NMP.

Normally, the computational D-M-R model concerns with four design domains, i.e. 1) requirement domain; 2) function domain; 3) meta-object domain; and, 4) form domain.

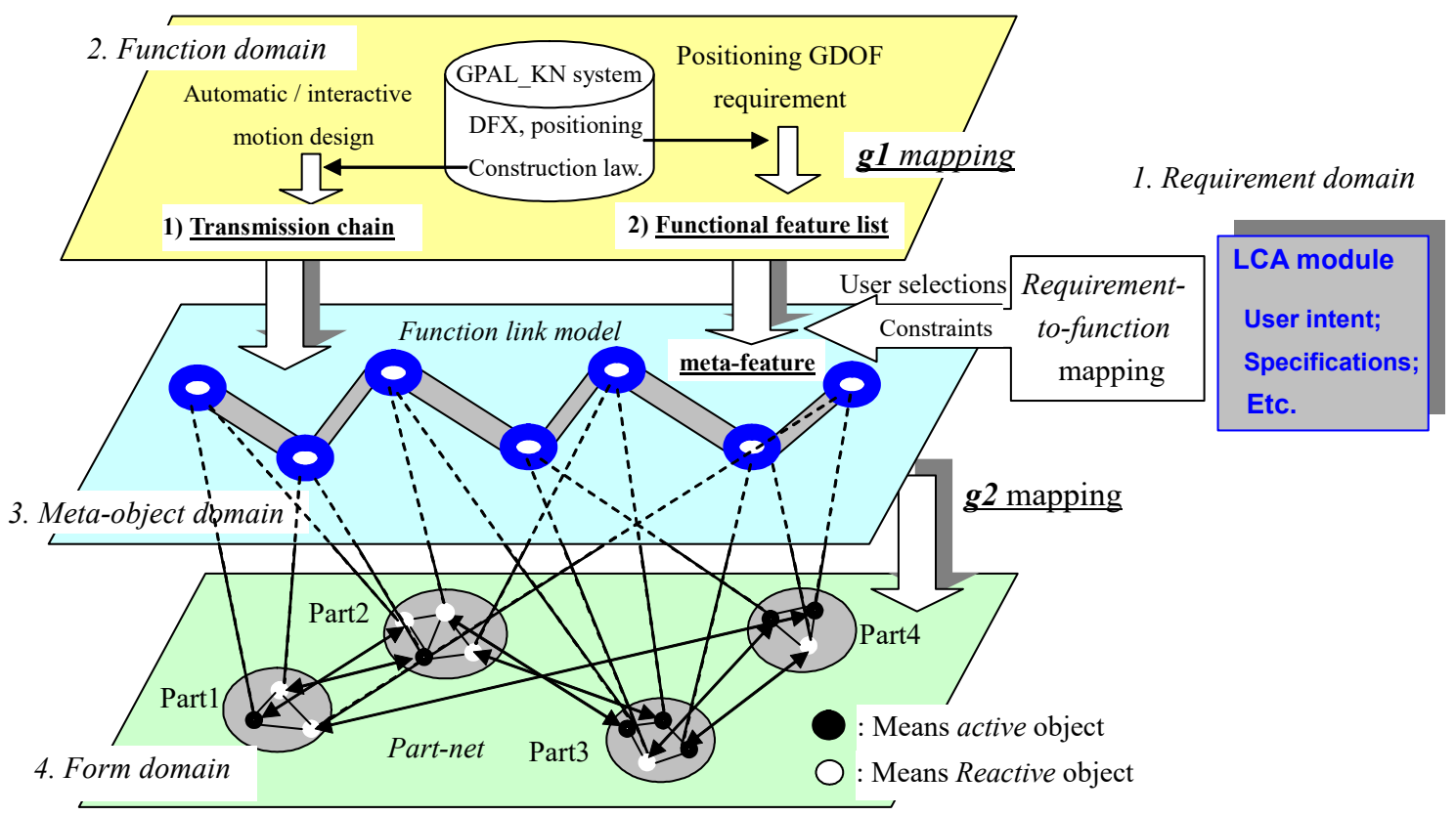

Figure 1. The D-M-R model

\section{D-M-R model in product design}

There are many decomposition and reconstitution (D\&R) models been proposed till now (Pahl and Beitz, 1996). Similar works are also found in the Function-Behaviour-State models. The proposed approach is a computational design management model, with automatic mapping schemes based on artificial intelligence; it is also an integrated methodology integrating conceptual and embodiment design; and a conceptual design tool exploiting default geometric reasoning and other mathematical algorithms to support conceptualization of design ideas.

\subsection{Introduction of D-M-R model}

The D-M-R model involves four major design domains, as in Figure 1: requirement domain, function domain, meta-object domain and form domain. And the engineering design is viewed as a mapping process from requirement domain to form domain. 
Firstly, the design domains associated with requirement, function and form are decomposed to specific granularity and in different graph styles, like a hierarchical tree in requirement domain, a parallel network structure in function domain or a hybrid model in the form domain, and the decomposition could help to set up domain specific knowledge base, mapping rules and criteria etc.

\section{Product level: like a lathe}

Performance/

Quality etc

Aesthetics/
Modelling/Ergonomics

Assembly/

Maintenance/

Disassembly Storage/Distribution

Recycling/

Reuse

Mechanism level: like the spindle system

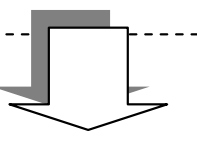

Transmission chain, mechanism draft, behavior set, simulation, analysis etc

DFA (Assembly) analysis,

DFD (Disassembly) analysis

Sequencing/Planning etc
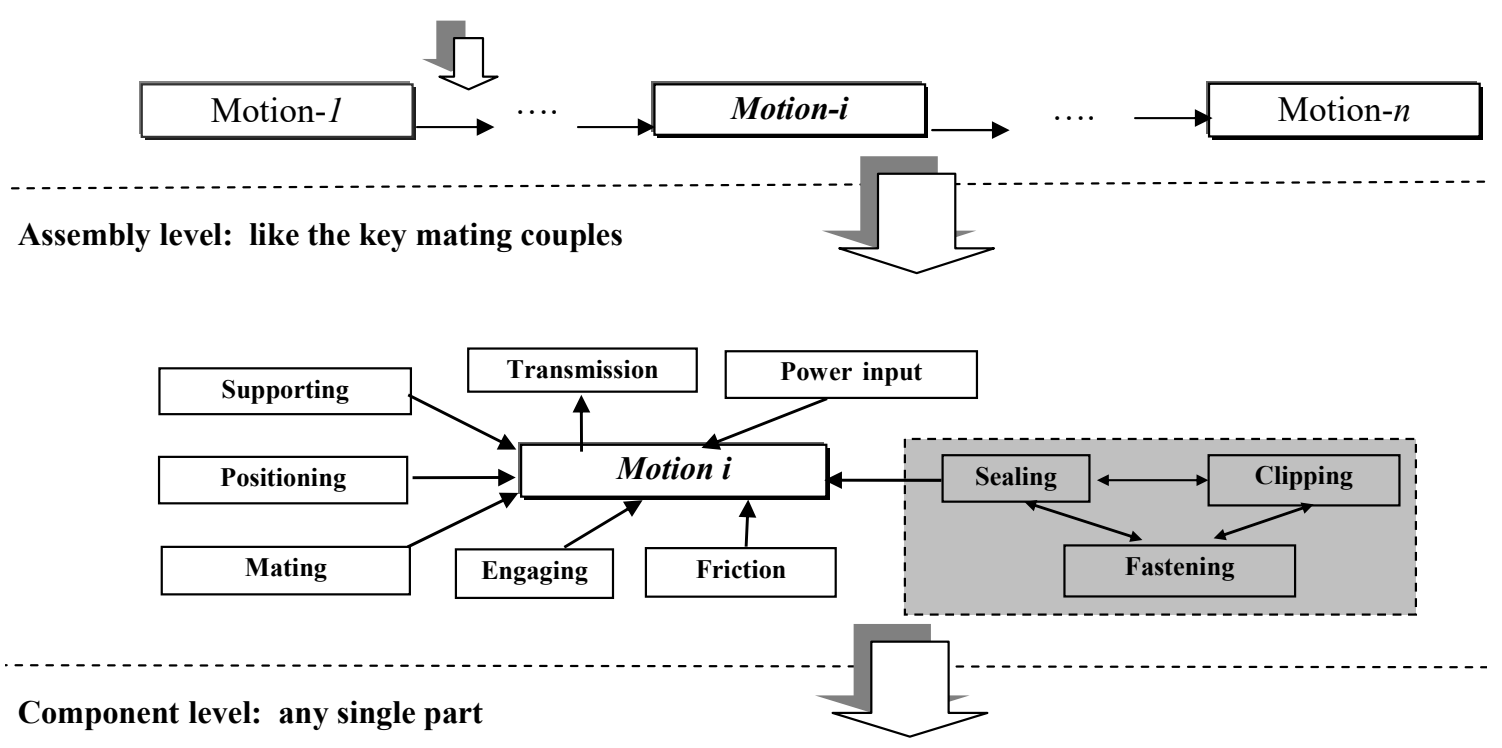

Manufacture / Demanufacture / Reuses / Analysis / Precision etc

Feature level: any single feature

Syntactic/ semantic implications within product life-cycle context.

Figure 2. Decomposition of function domain

Secondly, the mapping schemes are introduced ranging from requirement-to-function and function-toform etc. The mapping results are towards the form domain.

Lastly, the mapped discrete geometric and non-geometric elements in the form domain are synthesized with the help of specific knowledge base and default geometric reasoning. The following sections give the details.

\subsection{Function domain}

Functional domain is classified into three groups, quantitative, abstract, and assembly. We believe that functional definition is correlated to the problems concerned; product-level, assembly-level or 
component-level functions are to be taken into considerations. As long as the assembly structural design is concerned, the assembly level function is summarized, classified and analysed.

As in Figure 2, five levels of functional definitions are arranged in parallel rows, where the assemblylevel function is summarized as a connection link model, each node of which is an information unit to express the prerequisite assembly requirement, like positioning, transmission, supporting and lubrication in a network structure. The information unit could be correlated with many alternatives of functional carriers, what-ever it might be, a conceptual face, functional feature, component or mechanism etc.

\subsection{Meta-object domain}

One temporary design domain called meta-object domain is introduced in the F-F mapping model. The reason of the proposal of this domain is due to the gap between function and form. Actually, function and form belongs to different design domains, there are no natural connections between function connotations and geometric forms if no engineering implications are added to the physical forms.

In order to fill up the information gap, an interim domain, called the meta-object domain is introduced. The concept of meta-object is similar to a functional carrier, like an abstract feature corresponding to the kinematics requirement in F-F mapping. The meta-object could be a bridge of function and form domains.

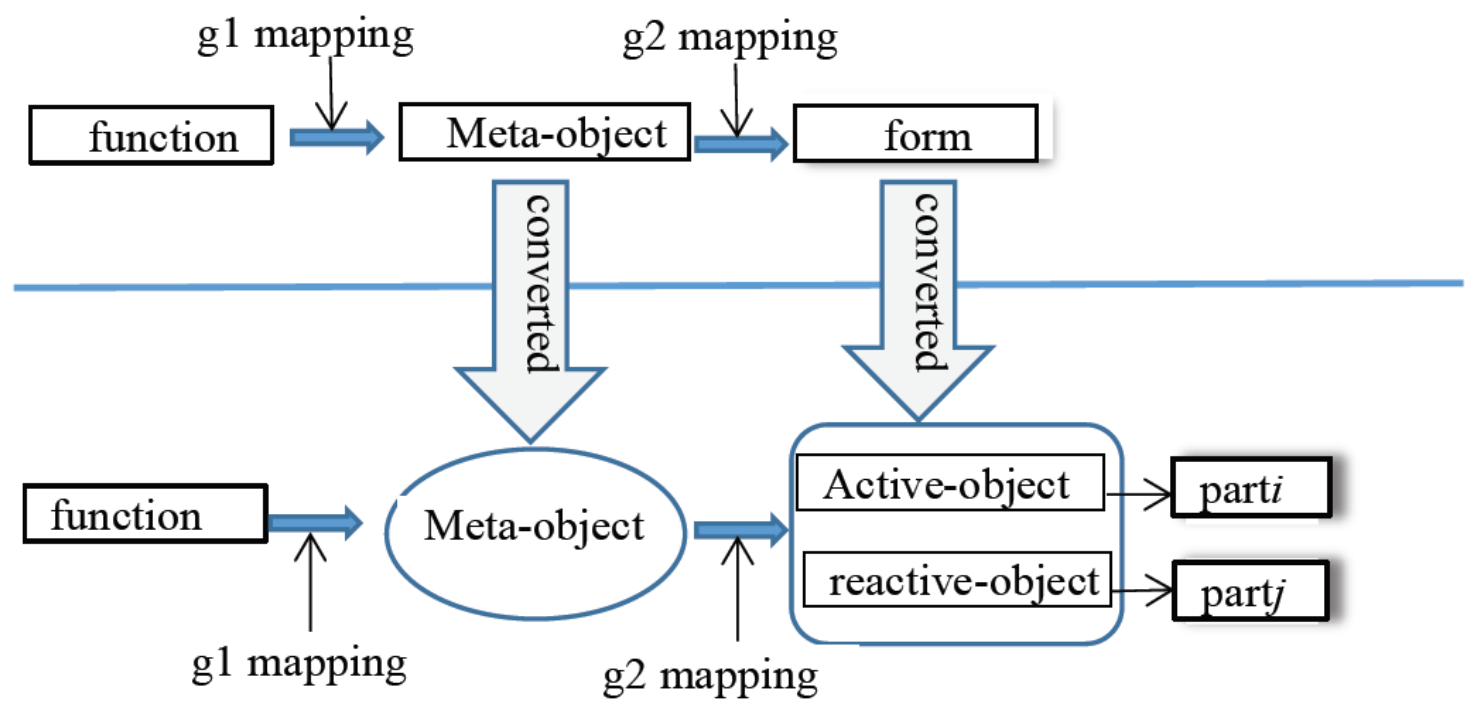

Figure 3. Function-form mapping process

In any one mechanism, there are pairs of objects, i.e. active-object and reactive-object, which belongs to different components, and corresponds to one meta-object.

The meta-object refers to the corresponding elements of a motion pair, active and re-active, and represents the concept of "functional carrier". In this way, the function is directly mapped to the metaobject, and the meta-object is mapped to the active and re-active object respectively, and the relationship between different components is established, like in Figure 3.

For example, if there is a positioning plane in a mechanism, there must be a corresponding orientation plane. Of course, the number, type, and size of the orientation surface and the orientation surface need not be consistent. The time validity of the existence of the action between the subject and the object is also different, this difference belongs to the attribute category of element.

Based on the viewpoint of action timeliness, there are dynamic orientation and static orientation between the active and reactive objects. 
Assembly level function establishes the relationship between parts, function-form mapping mainly refers to the mapping of assembly level function to 3D form of the product. It is actually divided into two processes: g1 and g2 mappings.

\section{4. g1 mapping}

The second-level creative support came from the function-to-form (F-F) mapping, which is called structural creative support, embedded in the following two mapping steps.

The first step function-to-form mapping is called g1 mapping: g1 mapping is to find suitable features for the corresponding kinematics requirement. It is the mapping process from the assembly-level function (Figure 2) to the functional features based on the kinematics positioning requirement of the component.

The results are dynamically stored in a list format. There exist many alternatives for a single component in one mechanism, so for a mechanism with large number of components, the combinational alternatives are intensively multiplied. There are two options: one is the potential to explore in a larger solution space, which is crucial for creative support; the other is the problem to cause combinational explosion and perturbations.

The Function-meta object mapping pattern is shown in Figure 4.

Assembly-level function

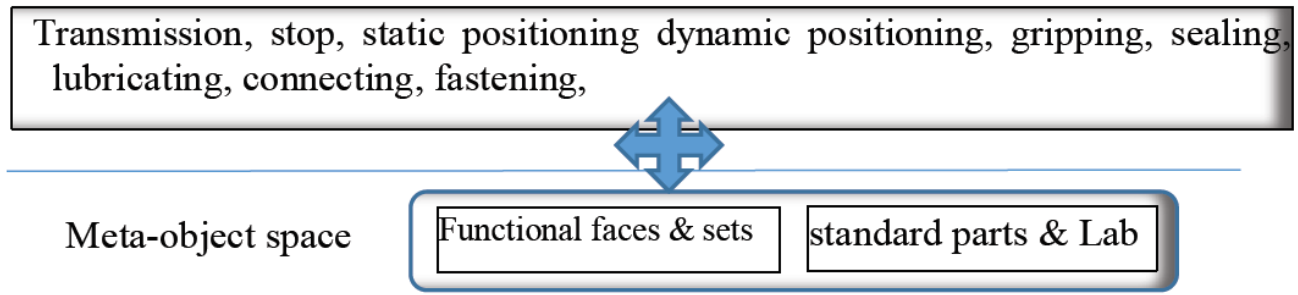

Figure 4. Function-meta object mapping

The function - meta object mapping is in a multi-multi-mapping pattern, so appropriate meta-object is selected according to different functional requirements. The Function- meta object mapping rules are as the follows.

- Lubricating - meta object mapping rules,

- Fixing - meta object mapping rules,

- Clamping -meta object mapping rules,

- Joining - meta object mapping rules,

- Fastening - meta object mapping rules,

- Sealing - meta object mapping rules.

If the functional surface (or functional surface cluster) is used as the basic decomposition space, all of the above functions can be mapped to the corresponding functional surface (or functional surface cluster). However, a large number of manuals have been formed, and the functions can be mapped directly to these standardized parts, this reduced the difficulty of entity reconfiguration; and the practicability of CAD system can be improved.

For example, lubrication functions are mapped to: oil cup + sealing ring + ring groove features. The fastening function is mapped to the element: thread seal + thread + plane.

\section{5. g2 mapping}

The second step function-to-form mapping is called g2 mapping: g2 mapping is the mapping process from functional features to product form. The functional features are actually the meta-objects. It is mapped to two corresponding elements belonging to parti and partj respectively in a mechanism. The 
g2 mapping process depends on the content and nature of such a function link model as in Figure 5, each node of which is a specific motion node indicating two correlated parts and the assembly properties, like positioning, transmission, supporting and lubrication in the network structure etc. The mapping steps are correlated closely to the requirement, function and form domains. The two-step F-F mapping served as the second level support for creativity, i.e. structural creativity.

After the g1 mapping process, the meta-object network model is formed after mapping the element to the assembly-level functional node is shown in Figure 5.

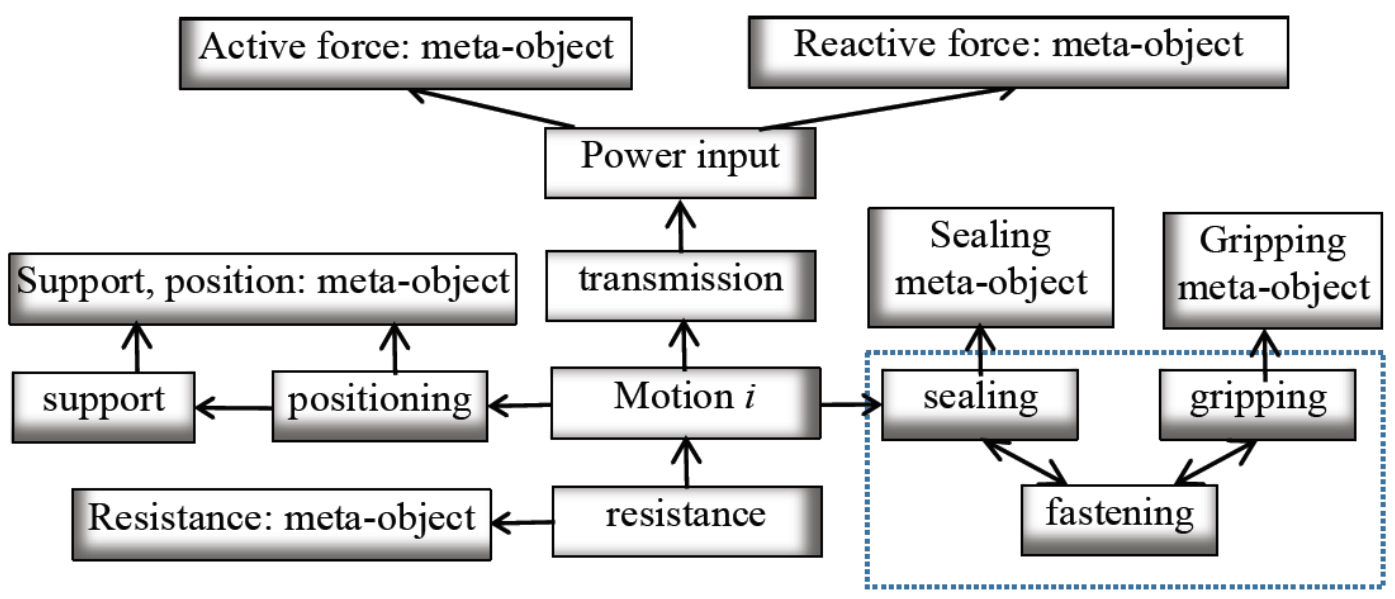

Figure 5. Meta object network model

Motion and force are the key factors of g2 mapping. In this case, motion is the relative movement between two parts, so a function motioni corresponds to two interrelated parts, i.e. PartAi and PartRi. PartAi refers to the actuator of the motion and forces; while partRi refers to the support of the said motion and force.

The mapping process from meta-object to part is actually the mapping process of functional carrier to the mechanism, that is, the process of creating conceptual structure of one assembly. It is actually the process of adding the active-objects and reactive-objects to the corresponding part and the correlation between the parts.

If the meta-object is planar object, the plane has the same positioning point and opposite normal vectors; and if the meta-object is cylinder object, the cylinder has the same diameter and opposite normal vectors. Based on the mapping of functional rotation surface and functional plane, the object has different positioning points and orthogonal vector directions.

PartAi plays a key role in the meta-object mapping process, where PartAi is associated with Container, PartRi, PartA(i-1) and PartA(i+1). The mapping process is shown in Figure 6.

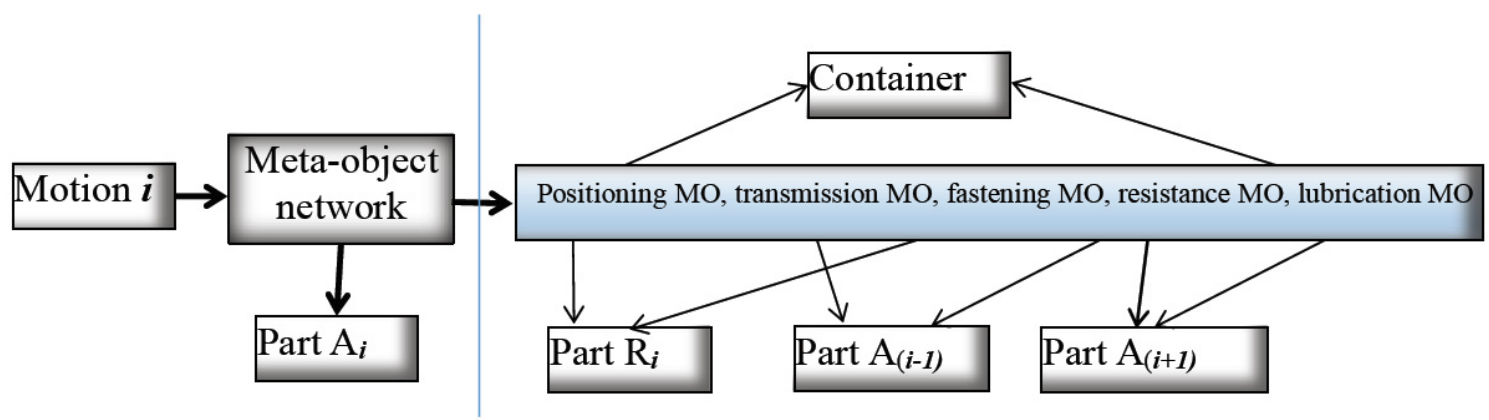

Figure 6. Meta object mapping model 
The meta-object in the network are all mapped to the PartAi as the active-object; and to the parts associated with the PartAi, as the reactive-object, like in Figure 7.

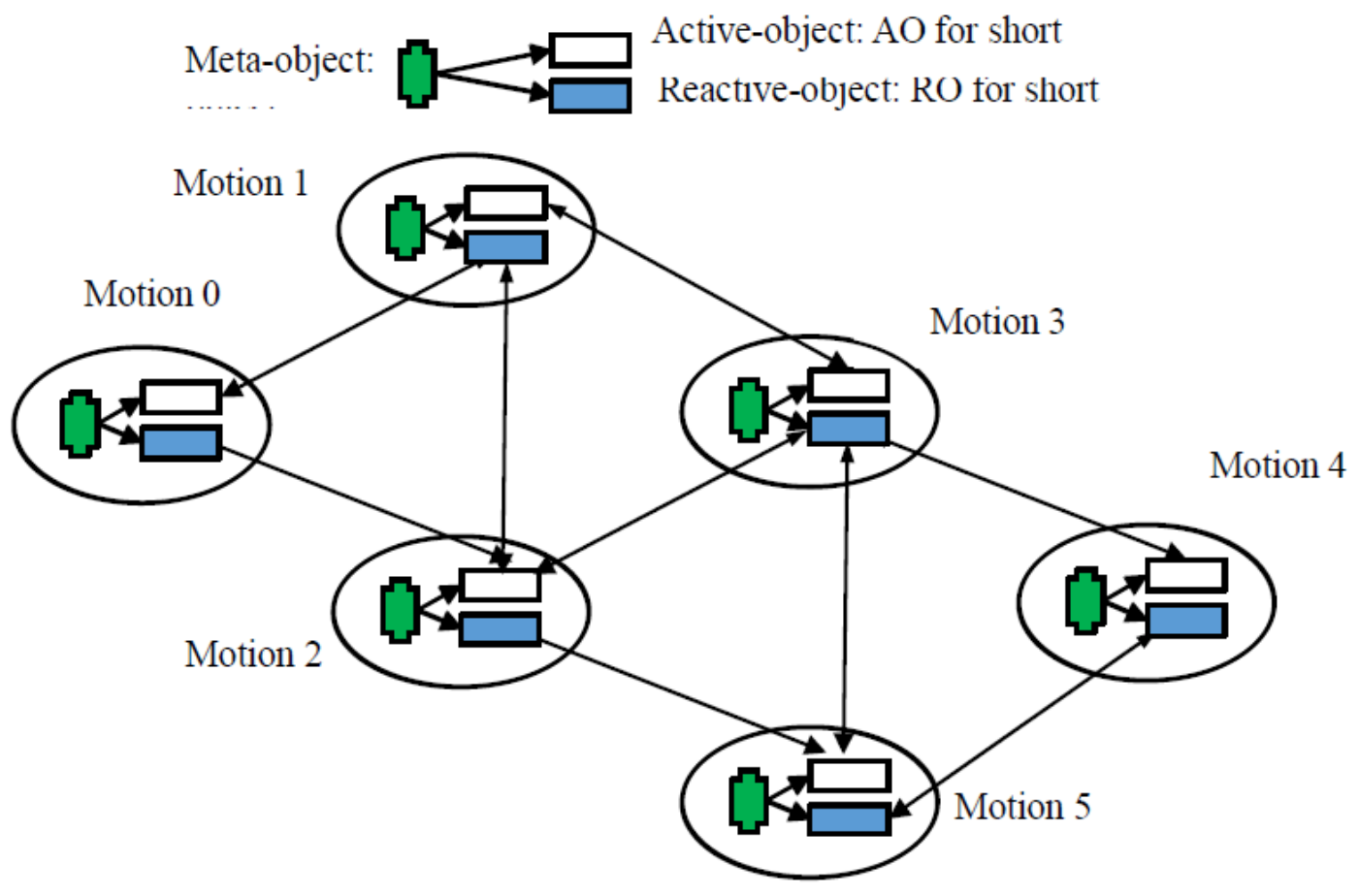

Figure 7. The network model of motioni

\section{Reconstitution of NMP}

Considering the importance of the early decision in the design process, the more difficult the decision is under the condition of incomplete information, the higher the value of the decision is. A series of problems, such as qualitative entity analysis and visualization of design process, have important research value.

In order to study the theoretical methods of geometric entity reconstruction under uncertain information, or incomplete information, this section draws on the default reasoning theory.

Default reasoning refers to the process of reasoning and decision making in the initial stage of a decision, when the information is incomplete or sometimes inaccurate, and some tentative assumptions are given. And carries on the theory aspect as well as the example aspect to verify.

Due to the absence of surface in conceptual entities and abstract features, a large number of auxiliary surfaces need to be added to the NMP in the process of entity reconstruction. The study shows that the ratio of the number of auxiliary surfaces added to the number of original functional surfaces is about $4: 1$.

Because of the complexity and diversity of the entity construction process, the geometric default reasoning process presents a divergent state. The default hypothesis condition is mainly imposed in order to converge the result of reconstruction within a certain range. The default reasoning theory [195] holds that the reasoning process does not always deduce the absolute from the correct premise, that is, the assumption condition. In the process of reasoning, it is often necessary to make certain hypotheses, which can be considered feasible provided there is no evidence to the contrary. In this paper, the following assumptions are made in the process of the entity default construction. Assumption 1: the refactoring principles for a single conceptual surface are as follows: 
A single concept plane, in its normal vector opposite and orthogonal direction, by default complements five orthogonal planes to form a hexahedron.

A single outer revolving surface, with two ends complemented by default.

A single inner surface, with a default complement of an outer surface and two ends.

Assumption 2: the refactoring principles for two conceptual planes are as follows

Two or more planes, which by default complement four orthogonal planes, form a hexahedron.

When the cylindrical surface intersects with the plane, a cylindrical end plane and four surrounding planes are complemented by default. The concrete reconstruction process should also depend on the concrete situation.

Assumption 3: the refactoring principles for the three conceptual planes are as follows

For three planes, the default complements three orthogonal planes to form a hexahedron.

When the cylindrical surface intersects with the other two planes, the number and position of the complement surface are considered in the position of the apparent plane.

Other situations should be analysed in detail as a result of specific problems.

The assumption of adding default surface in this section is also suitable for the reconstruction algorithm of abstract features. It is only a construction method which is more suitable for the design of mechanical parts, for example, it is more suitable for DFM, DFA requirements and so on.

In this paper, the following assumptions are made in the process of the entity default construction.

\section{Design examples}

A mechanical hand design example is to illustrate the design process and the creativity that involved. There are four major steps: 1) design requirement specification; 2) functional design; 3) F-F mapping for the embodiment design; 4) form reconstitution.

Step1. Design requirement specification.

The main functional requirement of the mechanical hand is to clamp the object, and to move the object from position1 to position2, like in Figure 8.

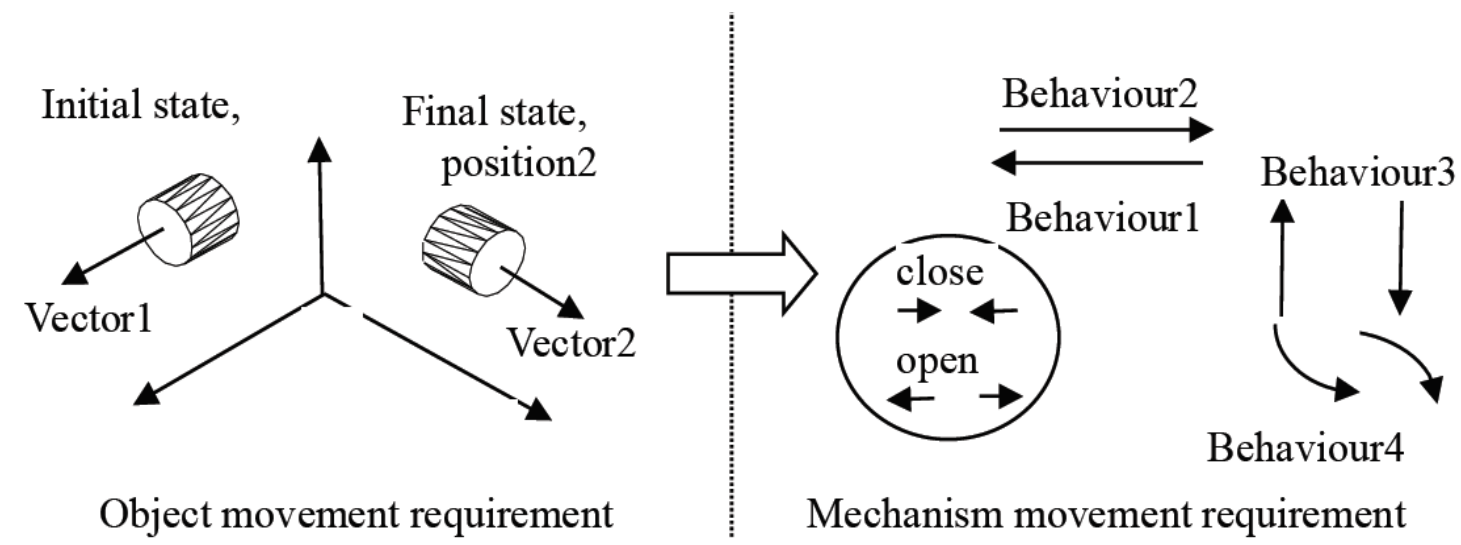

Figure 8. Design requirement of the mechanism

There are four movement requirements involved in the mechanism design (Table 2). The F-F mapping process falls into the following procedures.

Table 2. Behavior set of the mechanical system

\begin{tabular}{llll}
\hline Behaviour1 & Behaviour2 & Behaviour3 & Behaviour4 \\
\hline $\begin{array}{l}\text { Open and close } \\
\text { movement to clamp the } \\
\text { object }\end{array}$ & $\begin{array}{l}\text { Left and right } \\
\text { translation to move the } \\
\text { object }\end{array}$ & $\begin{array}{l}\text { Up and down } \\
\text { movement to pick up } \\
\text { or leave the object }\end{array}$ & $\begin{array}{l}\text { clockwise } \\
\text { counter-clockwise to change } \\
\text { position of the object }\end{array}$ \\
\hline
\end{tabular}


Step2. Functional design and creation of the computational transmission chain.

Combined with the right-hand diagram of Figure 8, the function structure of the transmission chain in the claw part can be deduced in Figure 9. In the schematic diagram, the components of the product structure are created at the same time.

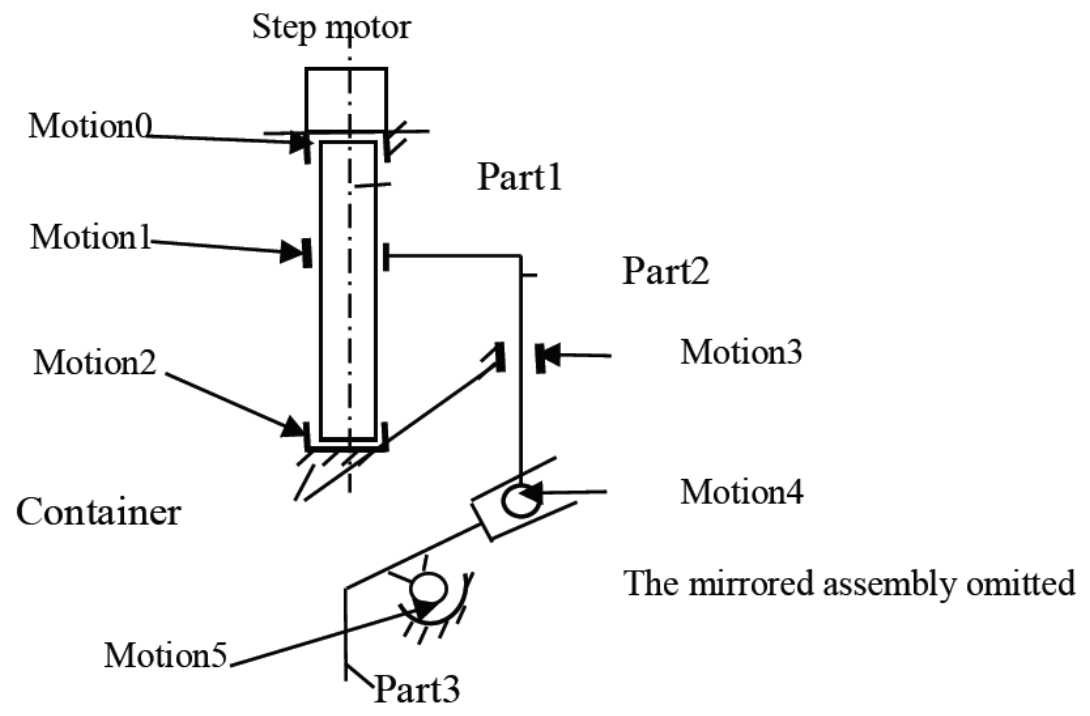

Figure 9. The computational transmission chain

Step3. Function-form mapping

Taking motion 0 as an example to realize a rotating motion function requirement, the element mapped to this rotational function requirement should include: 1 . The transmission element (receiving the power source of power source) and the positioning element (the component meeting the requirements of positioning and being positioned).

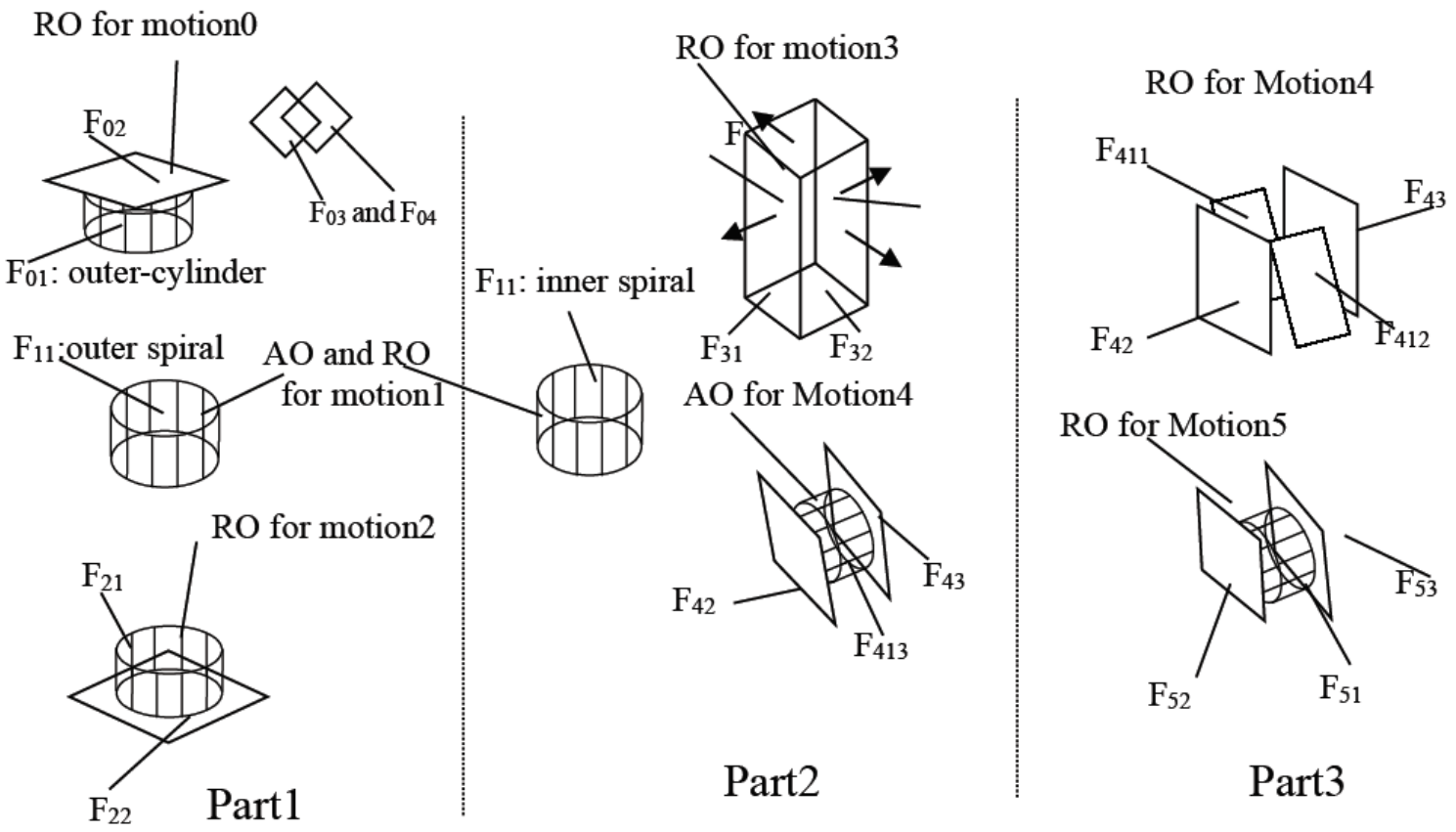

Figure 10. Function-form mapping of the transmission chain 
Transmission element: generally including positive and negative direction of the transmission requirements, so it should meet the two degrees of freedom of dynamic positioning. Considering the simple situation, $\mathrm{F}_{03}$ and $\mathrm{F}_{04}$ are taken as the transmission elements.

Positioning element: generally, except for rotational degrees of freedom, all other degrees of freedom of motion need to be eliminated, considering that the degrees of freedom in downward motion can be eliminated by the corresponding elements of motion2, so for motion0, it needs only to eliminate 9 GDOFs (Xu et al., 2002), and can choose a functional cylinder, called $\mathrm{F}_{01}$ and a functional plane, called $\mathrm{F}_{02}$.

According to the analysis method of motion0, the function-form mapping of motion1, motion2, motion2, motion3, motion4, motion5 can be realized, and the corresponding elements can be created, like in Figure 10.

The software has been developed and the top-down design paradigm is testified, like in Figure 11.

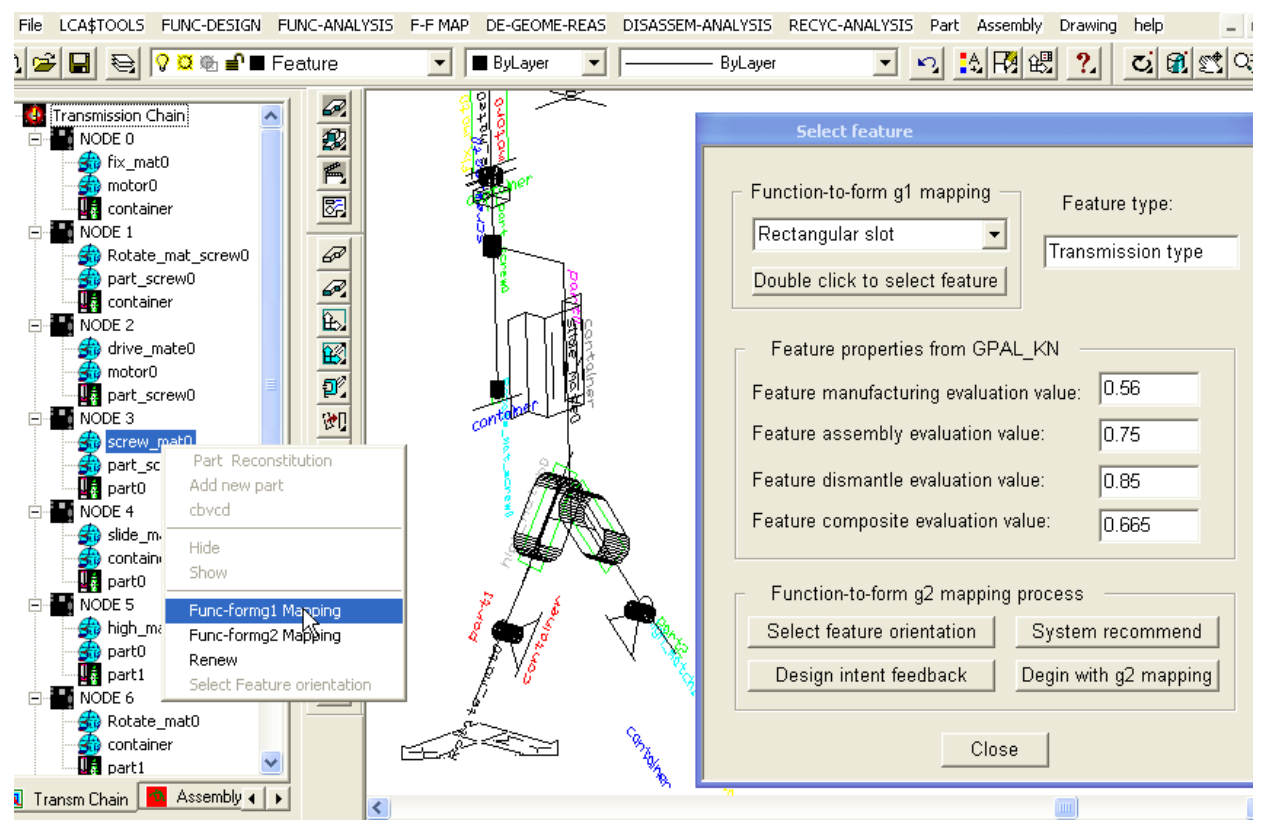

Figure 11. The clip part of the mechanical hand

\section{Discussion of the D-M-R model}

The proposed D-M-R model focused on highly automatic systems, like the automatic mechanism creation and automatic decomposition and synthesis. So human interactions, upper level requirement constrains with LCA inventories and domain specific knowledge base are introduced to guide the design process and avoid the combinational explosion of alternatives. The proposed D-M-R model is used for exploration in the design space, automatic transformation and convergent exploitation, which served as three levels of creativity support. The creativity support in product functional, structural and form is corresponded to the functional design, function-to-form mapping and form reconstitution respectively. Geometric reconstitution algorithm with the FF-NMP made it possible for the followings.

1. The D-M-R model is in the top-down style,

2. The design process is based on the NMP model,

3. The design process is from layout to part, so it is expected to realize the design efficiency of the complex mechanical assembly by providing the design guide and wizard tools like the software system such as word.

The 3D sketch design at assembly level begins with the 3D transmission chain of the assembly. The first definition is the assembly relationship between parts, which is equivalent to the whole to the detail, from the whole to the local. Therefore, the design process is top-down. 


\section{Conclusions and future works}

The presented study emphasized three points: decomposition, mapping and reconstitution (D-M-R for short), which is proposed to cope with divergent exploration, automatic transformation and convergent exploitation. Specifically, decomposition of design domains helps to set up the domain specific knowledge base (KB). Research on automatic mapping among design domains could dramatically increase exploration proficiency. And reconstitution algorithms involving default logic is to cope with ambiguous information occurred in conceptual design stage, which is helpful for conceptual visualization. The D-M-R model is also cooperated with the axiomatic design theory to make the design process applicable. This research is proved to be effective to integrate requirement, function and form domains for conceptual creative design.

\section{Acknowledgment}

The above-mentioned research work is supported by the Science, Technology and Innovation Commission of Shenzhen Municipality, JCYJ20160510165328965 and the Chinese NSFC, 61272017, P.R. China, and the National Demonstration Center for Experimental Mechanical Engineering Education (Shandong University).

\section{References}

Crocker, G.A. and Reinke, W.F. (1991), "An editable non-manifold boundary representation", IEEE Computer Graphics and Applications, Vol. 11 No. 2, pp. 39-51. https://doi.org/10.1109/38.75589

Darrell, L.M. (2003), "Better technology forecasting using systematic innovation methods", Technological Forecasting and Social Change, Vol. 70 No. 8, pp. 779-795. https://doi.org/10.1016/S0040-1625(02)00357-8

Dietz, P., Ort, A. and Penschke, S. (1998), "Classification of product knowledge - an approach to optimal feedback strategies for design", In: Jacucci, G., Olling, G.J., Preiss, K. and Wozny, M. (Eds.), Globalization of manufacturing in the digital communications era of the 21st century: Innovation, agility, and the virtual enterprise, Kluwer Academic Publishers, Dordrecht, pp. 783-799. https://doi.org/10.1007/978-0-387-353517_62

El-Mehalawi, M. and Miller, R.A. (2003), “A database system of mechanical components based on geometric and topological similarity. Part II: Indexing, retrieval, matching, and similarity assessment", Computer-Aided Design, Vol. 35 No. 1, pp. 95-105. https://doi.org/10.1016/S0010-4485(01)00178-6

Faltings, B. and Sun, K. (1996), "FAMING: Supporting innovative mechanism shape design", Computer-Aided Design, Vol. 28 No. 3, pp. 207-216. https://doi.org/10.1016/0010-4485(95)00027-5

Feng, C.X., Huang, C.C., Kusiak, A. and Li, P.G. (1996), "Representation of functions and features in detail design", Computer-Aided Design, Vol. 28 No. 12, pp. 961-971. https://doi.org/10.1016/0010-4485(96)00027-9

Pahl, G. and Beitz, W. (1996), Engineering design: A Systematic Approach, Springer Verlag, London. https://doi.org/10.1007/978-1-4471-3581-4

Xu, Z.-G., Frazer, J.H. and Tang, M.X. (2002), "Novel design methodology supporting product life-cycle design", Computers in Industry, Vol. 49 No. 3, pp. 253-265. https://doi.org/10.1016/S0166-3615(02)00100-8

Dr. Zhi-Gang Xu, Professor

Shandong University, Mechanical Engineering

17923, Jingshi Road, Lixiaqu, Wendongxiqu, Jinan, Shandong, 250061 Jinan, China

Email: zhgxu@sdu.edu.cn 